Max-Planck-Institut für demografische Forschung

Max Planck Institute for Demographic Research

Konrad-Zuse-Strasse 1 - D-18057 Rostock - GERMANY

Tel +49 (0) 3812081 - 0; Fax +49 (0) 3812081 - 202;

http://www.demogr.mpg.de

MPIDR WORKING PAPER WP 2010-015

FEBRUARY 2010

\title{
Cohort postponement and period measures
}

Joshua R. Goldstein (goldstein@demogr.mpg.de)

Thomas Cassidy (cassidy@demogr.mpg.de)

(C) Copyright is held by the authors.

Working papers of the Max Planck Institute for Demographic Research receive only limited review. Views or opinions expressed in working papers are attributable to the authors and do not necessarily reflect those of the Institute. 


\title{
COHORT POSTPONEMENT AND PERIOD MEASURES
}

\author{
February 26, 2010
}

\author{
JOSHUA R. GOLDSTEIN AND THOMAS CASSIDY \\ MAX PLANCK INSTITUTE FOR DEMOGRAPHIC RESEARCH
}

\begin{abstract}
We introduce a new class of models in which demographic behavior such as fertility is postponed by differing amounts depending only on cohort membership. We show how this model fits into a general framework of period and cohort postponement that includes the existing models in the literature, notably those of Bongaarts and Feeney and Kohler and Philipov. The cohort-based model shows the effects of cohort shifts on period fertility measures and provides an accompanying tempo-adjusted measure of period total fertility in the absence of observed shifts. Simulation reveals that when postponement is governed by cohorts, the cohort-based indicator outperforms the Bongaarts and Feeney model that is now in widespread use. The cohort-based model is applied to fertility in several modern populations.
\end{abstract}

\section{INTRODUCTION}

One way to view much of the demographic change that is taking place in advanced societies is as a result of the changing meaning of age. One often hears that 40 is the new 30 , or even 80 is the new 60. Demographers have developed formal models to show that shifting age-schedules (or equivalently, shifting meanings of age) can produce dramatic changes in cross-sectional period measures. Most notably, Bongaarts and Feeney's (1998) paper on fertility postponement, and their introduction of a "tempo-adjusted Total Fertility Rate" have become a fundamental part of the modern demographic toolkit.

The transformation of the human life cycle is a process that takes place within individual lives and is thus most naturally conceptualized as a cohort process. The magic of the Bongaarts and Feeney "tempo adjustment" is that only period data is needed. This is because postponement is modelled in period manner, with all ages (and thus all cohorts) postponing their events in the same manner in a given year. This rate of change can vary from period to period, but all cohorts must be treated the same. An enormous advantage of this approach apart from any degree of realism it may or may not have - is that it 
produces a wonderfully simple mathematical model, in which changes in period mean ages completely determine the presence and extent of tempo effects.

Criticisms have been levelled at the Bongaarts and Feeney (BF) approach. Notably, a number of authors have stated that the uniform postponement across all ages is unrealistic. However, few authors have shown the consequences of departure from this assumption, or have even proposed alternatives. Zeng Yi and Kenneth Land (2002) have shown, using a set of simulations, that violations of the uniform postponement by age assumption matter relatively little. Kohler and Philipov (2001) proposed a major extension to the BF approach, giving a fairly general framework for age and time varying postponement, and offering a special case in which postponement differed linearly by age within any period. The Kohler and Philipov results have not been widely used, in part because the paper is complex, the assumptions required to estimate the model are somewhat stylized, and the estimation procedure requires strong smoothing in order to produce interpretable results.

In this paper, we offer a conceptually simple alternative to the periodparamount view of postponement. Rather than uniform postponement by age within each period, we present a model in which there is uniform postponement in each cohort. Rodriguez (2006) provides inspiration by showing the relationship between these two views in the special case of linear shifts - that is when the rate of postponement (be it period or cohort) is unchanging over time. A simple version of our approach covers any trajectory of cohort postponement, with the important caveat that the same shift applies to all ages within a cohort. The mathematics of the model can be readily extended to include both variation in postponement by age within each cohort and also period effects on the level ("quantum") of fertility which introduce variable cohort quantum. We present procedures for estimating the simple version of the model and apply this to contemporary fertility patterns in several countries.

We begin by presenting the cohort shift model with period quantum effects in a simple form. We introduce an adjusted measure of period total fertility which can be used to recover the total fertility that would have been observed in the absence of postponement, and we use a few simple examples to illustrate how this adjusted measure relates to the observed period total fertility. Next we compare our cohort shift model with other measures of period total fertility. Finally, we show how the cohort shift can be estimated from data and apply our adjustment formula to real data to see how it compares to the BF adjustment. 


\section{Models of COHORT POSTPONEMENT}

Denote the fertility rate at age $a$ and time $t$ by $f(a, t)$. Translation from period to cohort is made using the fact that $t=c+a$, and so the fertility at age $a$ of the cohort born at time $c$ will be $f(a, c+a)$.

2.1. The cohort shift model. We let $\phi_{0}(a)$ denote a standard baseline fertility schedule that would have occurred had there been no postponement. In general, shifts in timing could vary by cohort and age, such that

$$
f(a, c+a)=\phi_{0}(a-S(a, c)) q(c+a),
$$

where $q(t)$ is a period change in the quantum of fertility and $S(a, c)$ is the "shift", which can vary by cohort and by age. For example, if "40" were the new "30" for the cohort of 1960 , then $S(40,1960)=10$.

A basic model of cohort postponement, which is still quite flexible in that it allows each cohort to postpone by a different amount, assumes uniform age-shifts within a given cohort. We let $S(c)$ be the total amount of shift experienced by cohort $c$. Thus

$$
f(a, c+a)=\phi_{0}(a-S(c)) q(c+a),
$$

or equivalently

$$
f(a, t)=\phi_{0}(a-S(t-a)) q(t) .
$$

For convenience we also assume that $\int \phi_{0}(a) d a=1$ where the unspecified limits of integration span all possible ages (this convention will be used throughout). Note that the period level effect $q(t)$ is invariant by age, and that the cohort shift effect $S(c)$ is invariant by period.

By definition the cohort total fertility rate (CTFR) for the cohort born in year $c$ is $C T F R(c)=\int f(a, c+a) d a$. Under this model the Cohort Total Fertility Rate depends on the history of period effects. If period quantum is constant over time, shifts within a cohort should not change the CTFR, and indeed we can see that this is the case. Replacing $f(a, c+a)$ with $\phi_{0}(a-S(c)) q$ gives us $C T F R(c)=\int \phi_{0}(a-$ $S(c)) q d a$. We evaluate this integral using the change of variables $w=$ $a-S(c)$ to get $C T F R(c)=q \int \phi_{0}(w) d w=q$.

The period TFR is influenced by both period quantum and the extent of cohort shifts. Writing

$$
T F R(t)=\int f(a, t) d a=\int \phi_{0}(a-S(t-a)) q(t) d a
$$

one can see that the sum of period fertility will depend on $S(t-a)$ Intuitively, this is because in the age-schedule in a given period will 
depend on the history of shifts for various cohorts. The degree to which the period TFR is influenced by cohort shifts will now be seen.

If the shifts $S$ are not constant, then conventional TFR will not be equal to the period effect $q(t)$. Notice that when we change variables in the expression $\int \phi_{0}(a-S(t-a)) q(t) d a$ by defining $w=a-S(t-a)$, we get $T F R(t)=q(t) \int \frac{\phi_{0}(w)}{1+S^{\prime}(t-a)} d w$, where $S^{\prime}(c)$ is the derivative of the shift function with respect to cohort. This calculation illustrates what is needed to recover the period effect $q(t)$ from $f(a, t)$. We therefore define a shift-adjusted period Total Fertility Rate as

$$
T F R^{\dagger}(t):=\int f(a, t)\left(1+S^{\prime}(t-a)\right) d a,
$$

so that $T F R^{\dagger}(t)=\int \phi_{0}(w) q(t) d w=q(t)$, and thus shift-adjusted $T F R^{\dagger}(t)$ recaptures the period TFR that would have been observed in the absence of cohort shifts.

The reason that the definition works is because the age-shifts from cohort-to-cohort are recapitulated in the cross-section from age-to-age. Increasing postponement effectively speeds up the clocks of those in a synthetic cohort within a given period. Likewise, slowing postponement means that the synthetic cohort within a given period will have more exposure at a given fertility rate. The neat thing about our adjustment is that rather than inflating or deflating the time spent at each age, we inflate or deflate the rate in a way that exactly compensates for the compression or extension of age introduced by the cohort shifts.

\subsection{Some simple examples.}

Estimation and application of the cohort shift model can be done by estimating $S^{\prime}(c)$ from data. However there are choices for $S(c)$ which give insight into the consequences of the cohort shift model. The following examples are useful to illustrate these consequences.

\section{Example 1: Linear shifts}

Following Rodriguez (2006), let $S^{\prime}(c)$ be a constant $k$. In this case, $T F R^{\dagger}$ is $(1+k)$ times the observed TFR. Since the fertility schedule $f(a, t)$ is $\phi_{0}(a-k t) q(t)$, period fertility is shifted but its shape is unchanged, and so the BF formula, $T F R^{*}=T F R /\left(1-\mu^{\prime}\right)$ is also applicable. It follows that $T F R /\left(1-\mu^{\prime}\right)=T F R(1+k)$ and so

$$
k=\frac{\mu^{\prime}}{1-\mu^{\prime}},
$$

which is the result obtain by Zeng and Land letting $r_{*}=k$ and $r=\mu^{\prime}$. 
Example 2: Polynomial shifts

Now consider the case that $S(c)$ is a polynomial in $c$. It is particularly interesting to center this polynomial at $-\mu(t)$ so that our cohort of interest is the one currently at the mean age of childbearing. So we can write $S(c)=\sum_{0}^{n} b_{i}(c+\mu(t))^{i}=\sum_{0}^{n} b_{i}\left(t-(a-\mu(t))^{i}\right.$ where the coefficients $b_{i}$ are constants. If our polynomial is quadratic then

$T F R^{\dagger}(t)=\int\left(1+b_{1}+2 b_{2} t-2 b_{2}(a-\mu(t))\right) f(a, t) d a=\left(1+b_{1}+2 b_{2} t\right) T F R(t)$ since the term $2 b_{2}(a-\mu(t))$ integrates to zero.

If the polynomial is cubic then $T F R^{\dagger}(t)$ is

$$
\begin{gathered}
\int\left(1+b_{1}+2 b_{2} t-2 b_{2}(a-\mu(t))+3 b_{3}\left(t^{2}-2 t\left(a-\mu(t)+(a-\mu(t))^{2}\right)\right) f(a, t) d a\right. \\
=\left(1+b_{1}+2 b_{2} t+3 b_{3}\left(t^{2}+\sigma^{2}(t)\right)\right) T F R(t)
\end{gathered}
$$

where $\sigma^{2}(t)$ is the variance of the schedule $f(a, t)$ with respect to $a$.

For polynomials of arbitrary degree we can use an approach that will work for any analytic function. Let $G(a)=S^{\prime}(t-a)$ and expand $G$ in a Taylor series about $\mu(t)$. Then

$$
\begin{gathered}
T F R^{\dagger}(t)=\int(1+G(a)) f(a, t) d a= \\
T F R(t)\left(1+S^{\prime}(t-\mu(t))+\sum_{n=2}(-1)^{n} \frac{S^{(n+1)}(t-\mu(t))}{n !} \mathfrak{K}(t)_{n}\right.
\end{gathered}
$$

where $\mathfrak{K}(t)_{n}$ is the centralized $n t h$ moment of the fertility schedule $f(a, t)$. If we expect the higher moments to be small, then we can use the following approximation

$$
T F R^{\dagger}(t) \cong\left(1+S^{\prime}(t-\mu(t))+S^{\prime \prime \prime}(t-\mu(t)) \sigma^{2} / 2\right) T F R(t) .
$$

\section{Comparison of $T F R^{\dagger}$ With OTher TEMPo-ADJUSTMENTS}

In this section we contrast $T F R^{\dagger}$ with other tempo-adjustments proposed in the literature.

\subsection{Comparison with Bongaarts-Feeney adjustment.}

Here we investigate the behavior of the BF adjustment procedure when applied to a setting in which postponement is purely cohort driven. We also show that the hypothetical postponement used in this comparison is not inconsistent with observed fertility rates.

Using simulations, we see that in periods of cohort shift transitions, $T F R^{\dagger}$ and the BF adjustment differ. In particular, if we postulate a rise in cohort postponement that spans multiple cohorts but eventually 
stabilizes, we observe the following pattern: when the $T F R$ begins to drop as a consequence of the onset of cohort postponement, the BF adjustment initially over compensates for the decline in $T F R$; when the cohorts with the greatest increase in postponement are at their peak fertility level, the BF adjustments underestimates the adjustment needed; as the end of postponement brings $T F R$ close to its original level the $\mathrm{BF}$ adjustment once again over shoots the ideal tempo-adjusted level.

To illustrate this phenomena we consider a highly stylized situation in which all women have exactly one birth, fertility is normally distributed and there is no period quantum change. Suppose also that postponement increases continuously from 0 to 3 years over the course of 30 as years as shown here.

Figure 1. A hypothetical history of cohort postponement

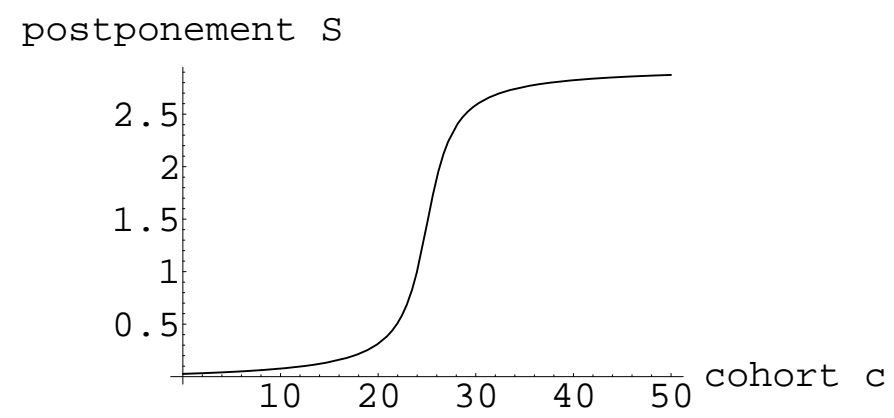

Under these assumptions we obtain the picture of $T F R, T F R^{\dagger}$ and $T F R^{*}$ shown in Figure 2.

During the 40 years in which postponement is changing the observed $T F R$, the BF adjustment both over and under compensates for this change. We also see that on average the BF adjustment is accurate over the long term. The short term volatility of the BF adjustment can be explained as a consequence of the age differences in the impact of cohort postponement on period measures. At the beginning of postponement the youngest cohorts experience a decline in fertility, and this has a larger influence on the mean age at birth than would a decrease of the same magnitude for cohorts who are currently closer to the mean age at birth. Similarly, as postponement comes to an end, only the oldest cohorts are undergoing a change in fertility, in this case an increase, and once again the effect on the mean age at birth is magnified.

Recent fertility in France provides an interesting case where this sort of postponement may in fact have occurred. Pla and Beaumel (2010) 
FiguRE 2. Simulated TFR, TFR and TFR*

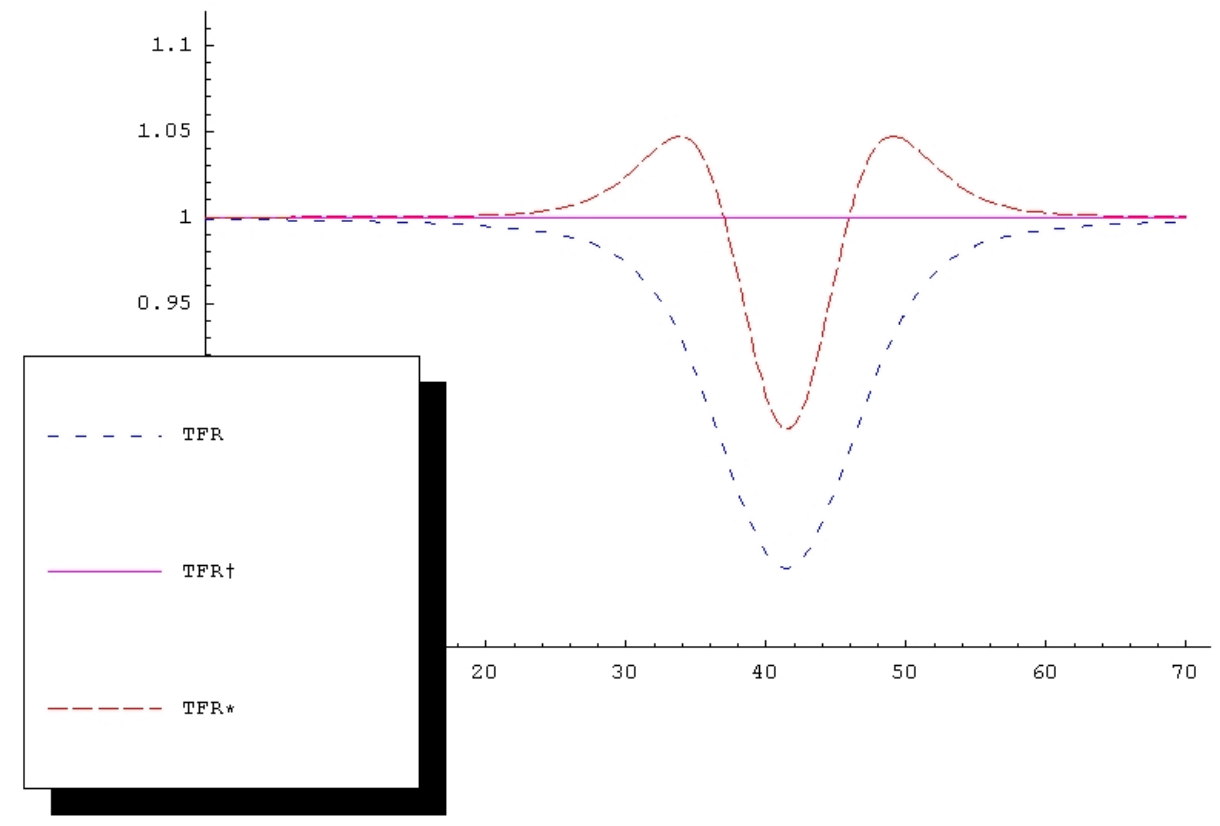

contrast period fertility schedules for French women in 1980, 1999 and 2009 to obtain the top picture in Figure 3.

Notice that fertility for women under the age of 25 changes from 1989 to 1999, but is virtually unchanged from 1999 to 2009, while for women over 27 the fertility curve continues to shift to the right. This could be explained as the outcome of cohort postponement which was increasing for the older cohorts but was ending for the cohort of 1975. A consequence of postponement stabilizing for the cohort of 1975 is that the younger women of 1999 and 2009 experience similar fertility rates while the older women do not. Indeed, when we model this using cohort postponement with a right skewed baseline fertility schedule and a postponement schedule that grows continuously from no postponement for the cohort of 1940 to four years of total postponement for the cohort of 1975, our simulation produces the lower picture in Figure 3.

While this similarity does not prove that cohort postponement has occurred, it does suggest that the cohort postponement model can be a viable explanation of observed changes in period fertility.

\subsection{Comparison with Average Cohort Fertility.}


FiguRE 3. Fertility in France and a cohort shift simulation nombre de naissances pour 100 femmes
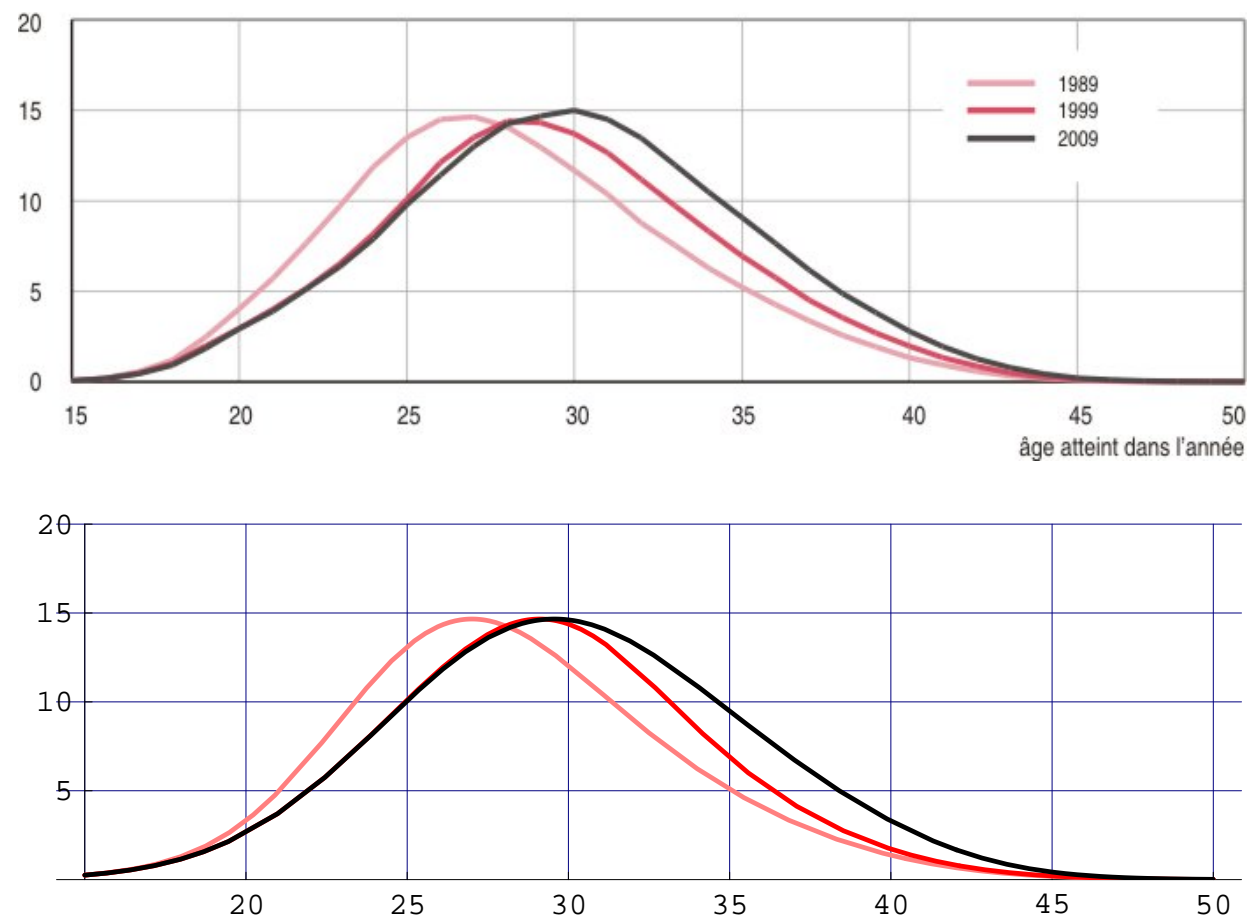

In order to contrast $T F R^{\dagger}$ with the Average Cohort Fertility (ACF) measure introduced by Butz and Ward (1979)(see also Schoen 2006), we consider a model in which cohort quantum, rather than period quantum, varies. This model can be summarized as

$$
f(a, t)=\phi_{0}(a-S(t-a)) q(t-a),
$$

where $q(t-a)$ is a factor which varies by cohort, but not by age. The interpretation of $T F R^{\dagger}$ in this case is as a weighted average of cohort fertility. The weights, $\left(1+S^{\prime}(t-a)\right) \phi_{0}(a-S(t-a))$, are a product of two factors. The first, tells us how to inflate the observed fertility in order to account for the cross-sectional compression of age that results from increasing postponement. The second tells us the fraction of cohort fertility occurring at age $a$, as a result of a shift in the baseline schedule.

The shift-adjusted total fertility rate in the case of varying cohort quantum gives a measure very similar to the ACF. That measure is

$$
A C F(t)=\frac{\int \beta(a, t) C T F R(t-a) d a}{\int \beta(a, t) d a},
$$


or in our notation,

$$
A C F(t)=\frac{\int \phi_{0}(a-S(t-a)) q(t-a) d a}{\int \phi_{0}(a-S(t-a)) d a} .
$$

The two measures are identical if, for example, cohort shifts are linear. In this case, the term $1+S^{\prime}(t-a)$ can be taken out of the integral as a constant and $\int f_{0}(a-S(t-a)) d a=1 /\left(1+S^{\prime}(t-a)\right)$, demonstrating the equality.

The two measures are also equal if cohort quantum is constant, with both measures equal to the constant level of cohort fertility.

In general, however, the two measures are not equal, since the weights need not have the same profile. We have found in simulation that ACF can differ from $T F R^{\dagger}$ considerably in the case of cohort shifts with period quantum. This is because a jump in quantum occurring in period $t_{0}$ will change the values of the weights $\beta(a, t)$ for all cohorts which experience fertility in year $t_{0}$, and consequently ACF will increase in years both before and after year $t_{0}$. In contrast, $T F R^{\dagger}$ will respond to this change in period quantum only in year $t_{0}$, and the value of $T F R^{\dagger}$ in adjacent years will be unaffected.

The important point here then is that $T F R^{\dagger}$ is a kind of moving average of cohort fertility, related but not necessarily identical, to ACF. Furthermore, the ACF measure can be reproduced exactly in cases where cohort fertility is constant, and/or shifts are linear. In these cases this gives us a new way to think of the ACF, not as some hypothetical measure that one could calculate if only one knew the completed fertility of all cohorts, but also as the measure that one would obtain by calculating $T F R^{\dagger}$ as the result of shift.

\subsection{Comparisons using a more general cohort shift model.}

Models of fertility in which postponement is a function of period, or of period and age, can be understood in the cohort shift framework if we employ the more general form of the shift function mentioned at the start of section 1 . Here we present a model of cohort shifts which allows for differing shifts within a cohort by age. We first describe this model and then show how it encompasses the models in the literature to date, notably that of Bongaarts and Feeney, Kohler and Philipov, and the cohort shift model presented above. ${ }^{1}$ The idea behind this more

\footnotetext{
${ }^{1}$ The way we conceive of postponement is as a kind of time and clock shifts. In order to avoid problems of shifts occurring to births that have already occurred and other logical inconsistencies, it is convenient to first allow time and age to shift as specified by the $S(a, c)$ function and then simply remap take a pre-assigned surface of births $B(a, c)$ and move them accordingly.
} 
general model is to define a function $u(a, t)$ that gives the incremental increase in postponement at age $a$ and time $t$. This, in combination with knowledge of the initial postponement of each cohort, allows a full description of any shift function $S(a, c)$ on the Lexis surface. ${ }^{2}$

Let $u(a, t)$ be the incremental increase in postponement at age $a$ and time $t$. Define the cumulative postponement for cohort $c$ by

$$
S(a, c)=\int_{0}^{a} u(x, x+c) d x+S(0, c) .
$$

where $S(0, c)$ is the "initial postponement" of cohort $c$. Note that partial derivative $S_{a}(a, c)$ is $u(a, a+c)$ and $S_{c}(a, c)=\int_{0}^{a} u_{t}(x, x+$ c) $d x+S_{c}(0, c)$.

To show how observed period values relate to the baseline cohort schedule, let $F_{0}(a)=\int_{0}^{a} \phi_{0}(x) d x$ be the cumulative fertility for the baseline cohort schedule. Following Rodriguez, let $F(a, c)=F_{0}(a-$ $S(a, c))$ be the cumulative fertility for cohort $c$ at age $a$ in the absence of any quantum effects. By differentiating $F_{0}(a-S(a, c))$ with respect to $a$ and then replacing $c$ with $t-a$ we get $f_{0}(a-S(a, t-a))\left(1-S_{a}(a, t-a)\right)$, which describes the fertility that would be observed at age $a$ in period $t$ if shifts were to occur without any quantum changes. The observed fertility rate $f(a, t)$ is then obtained by applying period quantum effects to these rates to produce

$$
f(a, t)=\phi_{0}(a-S(a, t-a))\left(1-S_{a}(a, t-a)\right) q(t) .
$$

Notice that if the period effects are constant, then $C T F R(c)$ is once again equal to $q$. The definition of $T F R^{\dagger}$ for this general version of cohort shifts is

$$
T F R^{\dagger}(t)=\int f(a, t) \frac{\left(1-S_{a}(a, t-a)+S_{c}(a, t-a)\right)}{1-S_{a}(a, t-a)} d a .
$$

To see why this definition works rewrite this expression by replacing $f(a, t)$ with $\phi_{0}(a-S(a, t-a))\left(1-S_{a}(a, t-a)\right) q(t)$ to get

$$
\int \phi_{0}\left(a-S(a, t-a)\left(1-S_{a}(a, t-a)+S_{c}(a, t-a)\right) q(t) d a .\right.
$$

Next set $w$ equal to $a-S(a, t-a)$ and change variables to get $\int \phi_{0}(w) q(t) d w=$ $q(t)$ so that once again $T F R^{\dagger}(t)$ is equal to the period quantum.

We now consider various examples of this more sophisticated model.

\footnotetext{
${ }^{2}$ Further generalization could treat each value $S(a, c)$ as the mean of some random variable, and an even further generalization could consider population mixing of distinct homogeneous populations.
} 
Example 1: The model presented in the first section is the special case that $u(a, t)=0$ and all postponement is determined by the initial postponement $S(0, c)$.

Example 2: Bongaarts and Feeney consider the situation in which the change in postponement is a function $r(t)$ of time which does not vary with age. This situation can be encompassed within our model by setting $u(a, t)=r(t)$ and $S(0, c)=\int_{0}^{c} r(x) d x$. Then $S_{c}(a, t-a)$ will be $r(t)$ and we get

$$
T F R^{\dagger}(t)=\int \frac{f(a, t)}{1-r(t)} d a=\frac{T F R(t)}{1-r(t)}
$$

which is exactly the result obtained by Bongaarts and Feeney.

Example 3: In order to investigate the consequences of variance effects in the BF formula, Philipov and Kohler consider a scenario in which cumulative postponement varies linearly with $a$. They choose a function of the form $S(a, t-a)=a-\bar{a}_{0}-\left(a-\bar{a}_{0}-\gamma t\right) e^{-\delta t}$ where $\gamma$ and $\delta$ are constants and $\bar{a}_{0}$ is the mean of the baseline schedule $\phi_{0}$.

Using Philipov and Kohler's form for $S(a, t-a)$ we find $S_{a}(a, t-a)=$ $1+e^{-\delta t}\left(-1+a \delta-a_{0} \delta+\gamma-\delta \gamma t\right), S(0, c)=a_{0} e^{-\delta c}-a_{0}+\gamma c e^{-\delta c}$ and $S_{c}(a, c)=e^{-\delta t}\left(a \delta-a_{0} \delta+\gamma-\delta \gamma t\right)$. Thus we calculate $T F R^{\dagger}(t)$ as

$$
\int \frac{f(a, t)}{1-\gamma-\delta\left(a-\bar{a}_{0}-\gamma t\right)} d a
$$

If $\delta=0$ then this is $(1-\gamma)^{-1} T F R(t)$. Otherwise, notice that since $\bar{a}_{0}$ is the mean of the baseline schedule, it follows that

$$
0=\int\left(w-a_{0}\right) f_{0}(w) d w=\int \frac{e^{-\delta t}\left(a-\bar{a}_{0}-\gamma t\right)}{1-\gamma-\delta\left(a-\bar{a}_{0}-\gamma t\right)} f(a, t) d a .
$$

Since $\delta \neq 0$ then we can factor $(1-\gamma) e^{-\delta t} \delta^{-1}$ out of the expression on the right to see that

$$
0=\int \frac{\delta\left(a-\bar{a}_{0}-\gamma t\right)}{(1-\gamma)\left(1-\gamma-\delta\left(a-\bar{a}_{0}-\gamma t\right)\right)} f(a, t) d a .
$$

Now via algebra we can write

$$
\begin{gathered}
T F R^{\dagger}(t)=\int \frac{f(a, t)}{1-\gamma-\delta\left(a-\bar{a}_{0}-\gamma t\right)} d a= \\
\int\left[\frac{1}{1-\gamma}+\frac{\delta\left(a-\bar{a}_{0}-\gamma t\right)}{(1-\gamma)\left(1-\gamma-\delta\left(a-\bar{a}_{0}-\gamma t\right)\right)}\right] f(a, t) d a
\end{gathered}
$$

which using equation 3 above is

$$
\int \frac{f(a, t)}{1-\gamma} d a=\frac{1}{1-\gamma} \operatorname{TFR}(t)
$$


and thus regardless of the value of $\delta$ we recover the Philipov and Kohler formula.

\section{Estimation of $T F R^{\dagger}$ With REAL DATA}

We return to the basic model of cohort shifts given in equation 1 . To estimate the shift-adjusted TFR, we need separate estimates of the adjustment factor $1+S^{\prime}(c)$ and of the period quantum $q(t)$. In practice we do not need to estimate either $S(c)$ or the baseline schedule $\phi_{0}$ since $T F R^{\dagger}$ is calculated from the observed fertility schedule $f$.

If we assume that the absolute value of $S^{\prime}$ is never greater than one, then period fertility recapitulates all the fertility rates experienced by cohorts, although the duration of exposure has changed. In this case, we can think of the adjustment factor $1+S^{\prime}(c)$ as a 'stretching' of aging so that the duration of period exposure is reset to match cohort exposure, and thus our period measure reproduces the cohort experience. In the absence of postponement, the derivatives of period fertility and cohort fertility with respect to age should be the same. With postponement, this ratio of derivatives measures the degree to which age has been compressed in a period, and consequently multiplying fertility rates by this ratio gives us the appropriate quantity to include in our adjusted total fertility rate. In mathematical terms this means that, in the absence of changing period quantum,

$$
1+S^{\prime}(c)=\frac{f_{a}(a, t)}{f_{a}(a, t)+f_{t}(a, t)}=\frac{\frac{d}{d a} f(a, t)}{\frac{d}{d a} f(a, c+a)} .
$$

One should be aware that both derivatives in this ratio will be zero at ages that correspond to peak levels of fertility, and therefore those ages cannot be used to calculate the adjustment factor. However, if postponement is a function of cohort only, then changing to a different age for the same cohort will provide an equally valid measure of the adjustment factor. Since in practice these derivatives can only be estimated, we have found it useful to calculate these values for multiple ages and then take the median of those values for each cohort. We use the median because the results of formula 4 are unstable for ages near peak fertility.

To see why the first equality in formula 4 holds notice that if $f(a, t)=$ $\phi_{0}(a-S(t-a)) q$ then $f_{a}(a, t)=\phi_{0}^{\prime}(a-S(t-a))\left(1+S^{\prime}(t-a)\right) q$ and $f_{t}(a, t)=-\phi_{0}^{\prime}(a-S(t-a)) S^{\prime}(t-a) q$. If in addition we allow period quantum to vary, then the role of $f_{t}$ in the denominator will need to be adjusted to compensate for the change in quantum over time. A 
FiguRE 4. Iterative estimates of cohort postponement and $T F R^{\dagger}$. This example uses Dutch fertility. Values are not smoothed.

Effect of iteration on estimates of cohort postponement (NLD)

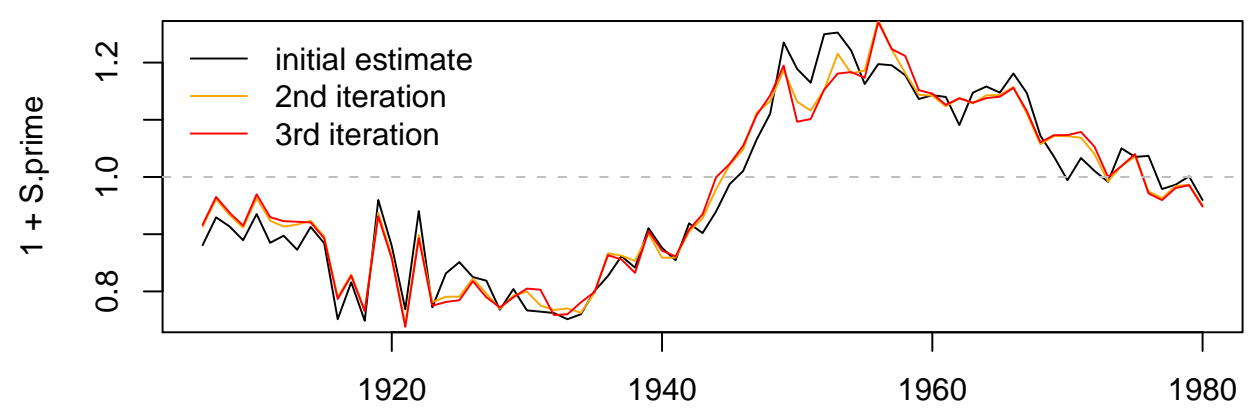

Cohort

Effect of iteration on estimates of TFR.dagger (NLD)

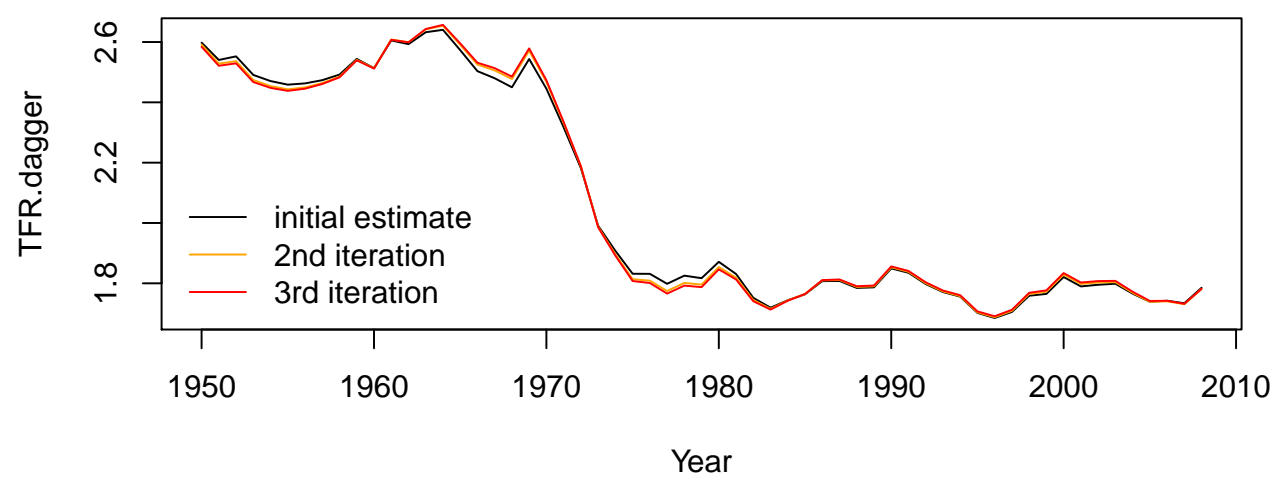

formula ${ }^{3}$ that captures $1+S^{\prime}(c)$ in the presence of changing period quantum is

$$
1+S^{\prime}(c)=\frac{f_{a}(a, t)}{f_{a}(a, t)+f_{t}(a, t)-f(a, t) q^{\prime}(t) / q(t)} .
$$

In an iterative calculation, the relative derivative $q^{\prime}(t)=q^{\prime}(t) / q(t)$ can be approximated by using formula 4 in a first estimation of $T F R^{\dagger}(t)$ and then substituting this value for $q(t)$ in to formula 5 . The result of

\footnotetext{
${ }^{3}$ Equations 4 and 5 are not the only formulas that extract $1+S^{\prime}$ from $f$. For example, the second derivative analog to equation 4 is $\left(f_{a a}+f_{a t}\right) /\left(f_{a a}+2 f_{a t}+f_{t t}\right)$.
} 
formula 5 can then be used to to get a new estimate of $T F R^{\dagger}(t)$, which in turn allows a new calculation of $1+S^{\prime}{ }^{4}$

To illustrate how the iterative procedure performs, we show the example of Dutch fertility in Figure 4. We see that the adjustment for period quantum given in equation 5 has a small impact on the measure of postponement for every cohort. It is apparent that the estimates of $1+S^{\prime}$ (and consequently $T F R^{\dagger}$ ) converge within just a few iterations. Indeed a fourth iteration of this process produces graphs that are indistinguishable from the third. We also note that the first estimate of $1+S^{\prime}$, using just formula 4 , is not very different from the quantum adjusted estimates, suggesting that formula 4 alone could be used to get an estimate of $T F R^{\dagger}$. $^{5}$

Figure 4 also illustrates the history of cohort postponement in the Netherlands. We see that the cohorts born before World War II, the parents of the babyboom, are in general advancing their fertility. The children of the babyboom generally postponed their fertility. For cohorts born after about 1970, however, no more postponement is detectable. The "postponement transition" seems to have come to an end (or at least a temporary one).

We now apply the cohort shift model to fertility data from the Netherlands, Sweden, the U.S.A. and Austria using the period life tables from the Human Fertility Database. In order to approximate the quantity $1+S^{\prime}(c)$ we have used equations 4 and 5 iteratively as described above. For purposes of comparison we also include the observed $T F R$ and $T F R^{*}$ in these illustrations. These pictures include two version of the BF adjustment. The first, labelled "period-tempo adjusted $T F R^{*}$," is calculated using the period mean age at birth for all births. The graph labelled "period-tempo adjusted, parity-specific, $T F R^{*}$ " was generated using the sum of the BF adjustments applied separately to each birth order as recommended by Bongaarts and Feeney. Our calculations for all these measures were limited to females between 15 and 45 years old. The results are presented in Figure 5.

In all four graphs we see common patterns. During the years when we might expect postponement to depress observed fertility rates (approximately from the mid 1970's into the 1990's), both the parity specific

\footnotetext{
${ }^{4}$ One can obtain a theoretically exact formula for the relative derivative $\dot{q}(t)$ in terms of derivatives of $f$. The fact that formula 5 gives the same values at $(a, t)$ and $(a-1, t-1)$ produces an equation relating $\dot{q}(t)$ and $\dot{q}(t-1)$. If we now change to the cohort born in year $t-a-1$ and equate the results of formula 5 evaluated at $(a, t-1)$ and $(a+1, t)$, we get a second equation relating $\dot{q}(t)$ and $\dot{q}(t-1)$. These two equations with two unknowns can then be solved to obtain the formula for $\dot{q}(t)$.

${ }^{5}$ All of the estimates we show in later figures use 5 iterations.
} 
Figure 5. TFR, TFR $R^{\dagger}$ and two versions of the BF adjustment in the Netherlands, Sweden, the U.S.A. and Austria
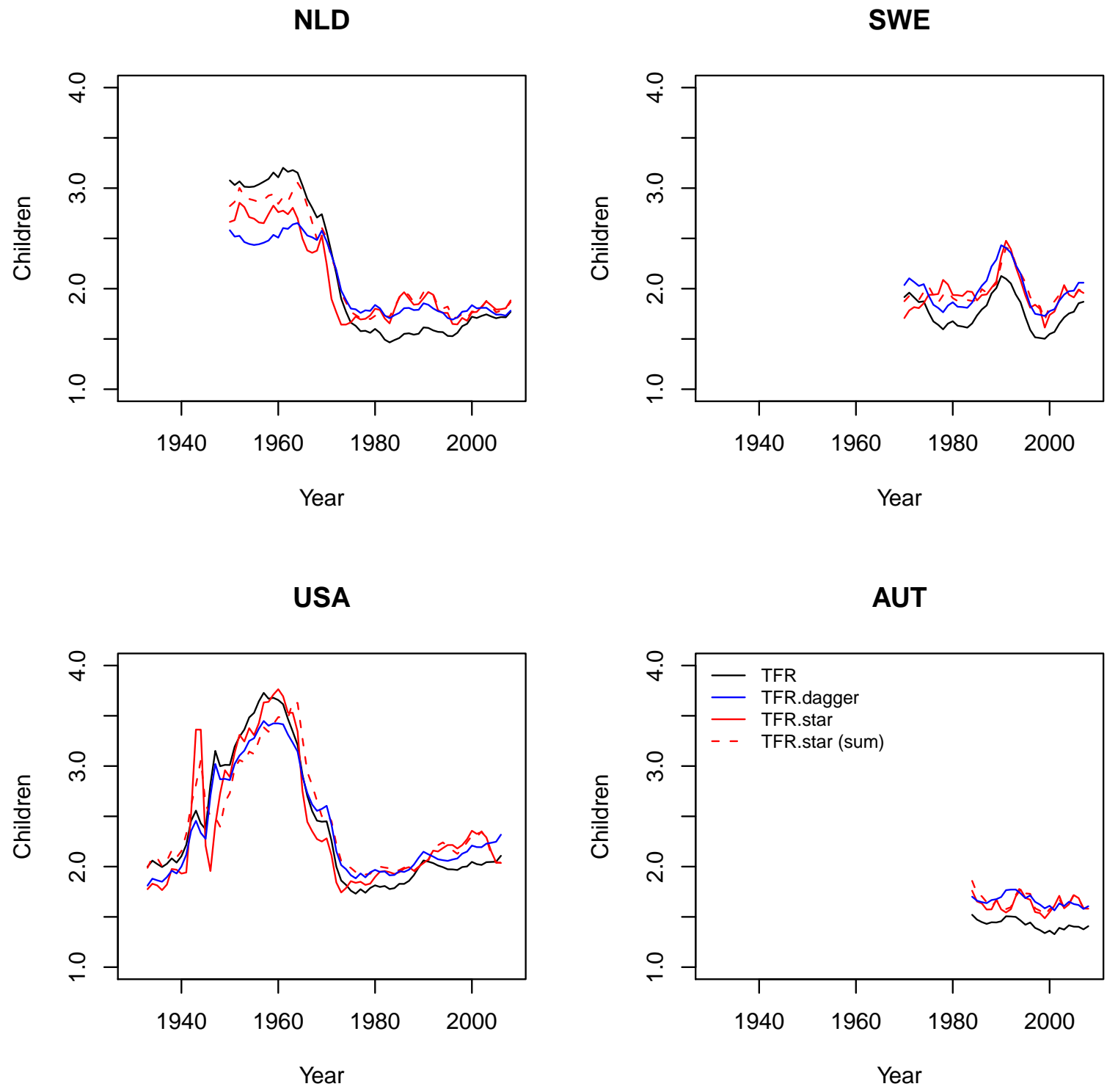

$T F R^{*}$ and $T F R^{\dagger}$ exceed $T F R$ and thereby compensate for the effects of postponement. The case of the United States is particularly illustrative. We see that during the late 1990's, when one would expect the postponement to be decelerating, the BF adjustments are higher than 
$T F R^{\dagger}$, just like in the simulation in section 2.1 (Figure 2). A similar phenomenon occurs for Holland and Austria. ${ }^{6}$ In all four illustrations we observe that $T F R^{\dagger}$ is comparatively smoother than $T F R^{*}$. This is what we would expect if shifts follow the cohort model given in equation 1, since $T F R^{\dagger}(t)$ effectively averages the shifts for all the cohorts experiencing fertility in period $t$. We also see that the fluctuations in $T F R^{\dagger}$ clearly parallel changes in the observed $T F R$, whereas the more dramatic jumps in $T F R^{*}$ are harder to interpret.

None of these illustrations can tell us whether postponement in these countries really does occur by cohort rather than by period. However these pictures make it clear that the adjusted total fertility rate given by $T F R^{\dagger}$ represents a plausible approach to addressing the distortions in period measures caused by postponement.

\section{Discussion}

The cohort shift model is at least an alternative formulation to the period perspective of Bongaarts and Feeney. At best, it will prove to be estimable and in some cases provide superior estimates of the underlying level of fertility.

The cohort-shift model fits into a larger class of shift models, which include all of the shift models we know of the literature to date. This general form for shift models does not - at least to us - present a full set of tractable analytic forms for the postponement function $u(a, t)$. However, it does, at least, allow us to understand how all of the different models to date relate to one another. In the future, it may also lead to useful flexible formulations of $u(a, t)$ that are both analytically understandable and behaviorally defensible.

\section{REFERENCES}

Bongaarts, J. and Feeney, G. (1998). On the quantum and tempo of fertility. Population and Development Review, 24(2):271-291.

Butz, W. P. and Ward, M. P. (1979). Will US fertility remain low? A new economic interpretation. Population and Development Review, 5(4):663-688.

Kohler, H. and Philipov, D. (2001). Variance effects in the BongaartsFeeney formula. Demography, 38(1):1-16.

Pla, A. and Beaumel, C. (2010). Bilan démographique 2009: Deux pacs pour trois mariages. Insee Premiere 1276:?

\footnotetext{
${ }^{6}$ A closer investigation of this phenomenon in Holland reveals that the jump in $T F R^{*}$ is the result of births at parities one and two.
} 
Rodríguez, G. Demographic translation and tempo effects: An accelerated failure time perspective. (2006). Demographic Research 14:85-110.

Schoen, R. 2006. Dynamic Population Models, Springer.

Zeng Yi and Land, K.C. (2001). A sensitivity analysis of the BongaartsFeeney method for adjusting bias in observed period total fertility rates. Demography, 28:17-28.

Zeng Yi and Land, K.C. (2002). Adjusting period tempo changes with an extension of Ryder's basic translation equation. Demography, 39(2):269-285. 